\title{
Impact of supplemental training programs on improving medical students' confidence in providing diabetes self-management education and support
}

\author{
This article was published in the following Dove Press journal: \\ Advances in Medical Education and Practice \\ 28 September 2017 \\ Number of times this article has been viewed
}

\author{
Maryam T Fazel ${ }^{1,2}$ \\ Mohammad Fazel ${ }^{3}$ \\ Nora L Bedrossian ${ }^{3}$ \\ Fernando Picazo ${ }^{3}$ \\ Julia D Sobel ${ }^{3}$ \\ Mahdieh Fazel ${ }^{3}$ \\ Charisse $\mathrm{Te}^{2}$ \\ Merri L Pendergrass ${ }^{2}$ \\ 'Department of Pharmacy Practice \\ and Science, College of Pharmacy; \\ ${ }^{2}$ Department of Medicine, Division of \\ Endocrinology, College of Medicine - \\ Tucson, ${ }^{3}$ College of Medicine - Tucson, \\ The University of Arizona, Tucson, \\ AZ, USA
}

Background/purpose: The purpose of this study was to evaluate the effectiveness of supplemental diabetes-related training modalities and volunteer activities in increasing first-year medical students' knowledge/comfort in providing diabetes self-management education and support (DSMES) to patients.

Methods: A group of medical students developed supplemental diabetes-related training/ volunteer programs. The training modalities included an optional 7-session interprofessionally taught Diabetes Enrichment Elective and a 3-hour endocrinologist-led training session intended to prepare students for involvement in an inpatient DSMES volunteer program. The volunteer program provided the students with the opportunity to provide DSMES to patients with diabetes admitted to an academic medical center. Those participating in any of the stated programs were compared to those with no such training regarding confidence in providing DSMES using an optional online survey. The results were analyzed by using Mann-Whitney $U$ test and descriptive analyses.

Results: A total of 18 first-year medical students responded to the optional survey with a response rate of $\sim 30 \%$ (10 of 33) among participants in any training/volunteer program. First-year medical students who attended any of the offered optional programs had statistically significant higher comfort level in 4 of the 6 areas assessed regarding providing DSMES compared with those with no such training $(p<0.05)$, with medium to large effect size $(r=0.48-0.59)$.

Conclusion: This study suggests that the supplemental preclerkship diabetes-specific training modalities/volunteer programs can provide benefit in providing medical students with practical knowledge while improving their confidence in providing DSMES to patients with diabetes.

Keywords: preclerkship, interprofessional, volunteer, training, practical, elective, confidence

\section{Background}

Diabetes mellitus is a major health concern worldwide with increasing prevalence. ${ }^{1}$ Approximately 29.1 million of US population is affected by diabetes. ${ }^{2,3}$ The high prevalence of diabetes and its associated complications including cardiovascular disease, nephropathy, blindness, neuropathy, and lower extremity amputations substantiate the burden of the disease. ${ }^{4}$ Hospitalizations and hospital stay days are increased among patients with diabetes when compared with patients without diabetes. ${ }^{5}$ Approximately $20 \%$ of all hospitalizations in the US in 2008 involved patients with diabetes regardless of admission diagnosis. ${ }^{6}$ Furthermore, patients with diabetes had longer and more costly hospital stays. ${ }^{6}$ Hospital discharges in 2014 included 7.2 million adults with
Correspondence: Maryam T Fazel College of Pharmacy, The University of Arizona, 1295 N. Martin Ave. (Room B2IIB), Tucson, AZ 8572I-0202, USA

Tel +I 520626429 I

Fax +I 5206267355

Email maryamfazel@pharmacy.arizona.edu 
diabetes as a listed diagnosis. ${ }^{7}$ In a 2016 position statement by the American Association of Diabetes Educators (AADE), the importance of diabetes self-management education and support (DSMES) in improving patient care and recovery and reducing hospital costs was emphasized. ${ }^{8}$

The AADE recommends that all inpatient teams include a diabetes educator to guide and support patients and their families on diabetes self-management during diabetes- or hyperglycemia-related hospitalizations. ${ }^{8}$ The 2017 National Standards for DSMES highlight the importance, demonstrated benefits, as well as underutilization of DSMES in diabetes care. ${ }^{9}$

An article by Nettles stated that healthcare providers can utilize accessibility to hospitalized patients with diabetes as an opportunity to further educate them regarding diabetes self-management. ${ }^{10}$ Institutions that executed this demonstrated a decrease in all-cause hospital readmissions within 30 days, an improvement in medication adherence after discharge, lower hemoglobin A1C 3-6 months after discharge, and better glycemic control 1 year after discharge in patients newly discharged on insulin. ${ }^{11-14}$ These outcomes are correlated with both improved quality of life and decreased healthcare utilization and costs, further demonstrating the importance of DSMES through the availability of knowledgeable inpatient diabetes educators. ${ }^{15-17}$ A pilot study showed that diabetes education during hospital stay has the potential to improve blood glucose and treatment satisfaction. ${ }^{18}$

Medical students are in prime position to provide such education, but studies suggest that further training is necessary to adequately provide these services in the inpatient setting. Shahla et al used a questionnaire to assess medical professional and medical student knowledge regarding diabetes. This study demonstrated that there was room for improvement in knowledge base among all groups assessed with medical students having the lowest scores, an average of $59 \%$ of questions answered correctly. ${ }^{19}$ George et al demonstrated trainee doctors' lack of confidence in the management of diabetes and that $>70 \%$ of participants required additional training in this area. ${ }^{20}$ In addition, a study by Lansang and Harrell demonstrated that fourth-year medical students had inadequate knowledge of inpatient diabetes management and suggested designing a curriculum to facilitate training to teach this information. ${ }^{21}$

In a previous study by our group, the first- and secondyear medical students' confidence in specific areas related to providing DSMES significantly increased following participation in the preclerkship diabetes-specific optional course
(Diabetes Enrichment Elective). ${ }^{22}$ Our current study is to expand the previous study results by discussing qualitative effectiveness of various training modalities implemented including participation in a preclerkship optional elective in diabetes mellitus and/or in a 3-hour endocrinologist-led inpatient DSMES training with or without involvement in related inpatient volunteer activities on improving medical student confidence in providing DSMES to hospitalized patients with diabetes when compared to students who did not receive such training.

\section{Methods}

Students enrolled in the College of Medicine - Tucson, the University of Arizona, have the opportunity to develop and/ or participate in community service groups under the Commitment to Underserved People (CUP) program. A diabetesfocused CUP program was developed by three medical students in 2015 entitled Diabetes Education, Prevention, and Outreach (DEPO). In an attempt to supplement fellow medical students' practical knowledge and comfort with regard to discussing diabetes self-management with patients, various educational/training programs as well as an inpatient volunteer program were developed by the DEPO CUP program.

The educational/training programs developed included an optional 7-session Diabetes Enrichment Elective taught by an interprofessional team of educators including pharmacists, dietitians, endocrinologists, and nurses. The suggested curriculum outlined by Standard 6 of the American Diabetes Assosiation National Standards for DSMES was used as a guide for the topics covered in this enrichment elective. ${ }^{23}$ The sessions provided included information regarding diabetes overview and medications, insulin pump therapy, proper insulin administration/use, exercise/fitness, and healthy eating (Table 1). The process of developing this optional enrichment elective is detailed in a previous article. ${ }^{22}$

A second training program was developed in order to prepare students to participate in inpatient DSMES volunteer program. This training program was a 3-hour endocrinologistled session that included education on various aspects of DSMES including the management of hyperglycemia/hypoglycemia, sick day diabetes management, physical activity, eating habits, blood glucose monitoring, and various available patient education resources. Those students who completed the 3-hour endocrinologist-led training session were provided the opportunity to participate in inpatient DSMES volunteer program/activity at Banner - University Medical Center Tucson, an academic medical center. This volunteer activ- 
Table I Diabetes enrichment elective schedule

\begin{tabular}{lll}
\hline & Session title & Instructor(s) \\
\hline Week I & Overview of diabetes & Endocrinologist \\
Week 2 & Diabetes prevention & Endocrinologist \\
Week 3 & Diabetes and fitness/exercise: why is exercise good for diabetes & Endocrinologist \\
Week 4 & Overview of diabetes medications & Pharmacist-certified diabetes educator \\
Week 5 & Proper insulin injection technique, mixing, and storage/blood sugar self-testing & Pharmacist- and nurse-certified diabetes \\
Week 6 & Insulin pump therapy overview & educators \\
Week 7 & Diabetes nutrition and healthy eating & Pharmacist-certified diabetes educator \\
\hline
\end{tabular}

ity consisted of discussing diabetes self-management with patients with diabetes regardless of their admission diagnosis. This provided the students with the opportunity to have oneon-one interactions with patients with diabetes in the inpatient setting and use the knowledge/skills they had gained in order to assist patients in a clinical setting.

In our previous study, we evaluated the effectiveness of the Diabetes Enrichment Elective during its first year of implementation using a retrospective pre- and post-course 5-question survey of first- and second-year medical student attendees. ${ }^{22}$ Upon implementation of this enrichment elective and in order to evaluate various training modalities further, a different methodology in the form of comparison with a control group was used. In doing so, first-year medical students who completed the optional Diabetes Enrichment Elective, completed the inpatient DSMES volunteering training session (with the opportunity to subsequently participate in the diabetes inpatient volunteer program), or had no such training were emailed an anonymous and optional survey. The survey included demographic questions regarding age/sex, questions regarding students' participation in various DEPO training/ volunteer programs, and a 6-question survey. The survey was preceded by a consent form providing information about the survey and that the completion of the survey was deemed to be agreement of consent.

The questions in the 6-question survey were intended to assess the students' comfort with regard to DSMES knowledge and the ability to discuss such information with patients. In doing so, the results of the survey were intended to assess the efficacy of various DEPO training/educational programs as well as the diabetes inpatient volunteer program in increasing first-year medical students' comfort in discussing diabetes self-management with patients as compared to those receiving no such training. A 5-point Likert scale was used for responding to the 6-question survey. Mann-Whitney $U$ test and descriptive analyses were utilized to assess the results of the survey. The study was approved by the Institutional Review Board of the University of Arizona.

\section{Results}

A total of 18 ( 8 female and 10 male) first-year medical students responded to the survey, with 8 of these students having received no DEPO training/education (Table 2). Of those who received some form of DEPO training/education (with some having also participated in the inpatient DSMES volunteer program), $30 \%$ ( 10 of 33 ) of students responded to the emailed survey. Of the 10 respondents who participated in some form of DEPO training/educational program, two (20\%) students participated only in the Diabetes Enrichment Elective, three $(30 \%)$ students participated in the Diabetes Enrichment Elective as well as the 3-hour diabetes inpatient volunteering training, three $(30 \%)$ students participated in the 3-hour diabetes inpatient volunteering training as well as volunteering within the program, and two $(20 \%)$ students participated in all three programs (ie, Diabetes Enrichment Elective, 3-hour diabetes inpatient volunteering training, and volunteering within the inpatient program). The majority $(86 \%)$ of respondents who participated in the Diabetes Enrichment Elective attended three or more sessions. All 5 $(100 \%)$ of those who participated in the inpatient volunteer program volunteered three or more times prior to the completion of the survey.

The 6-question survey results of first-year medical students who had participated in any form of DEPO DSMES training

Table 2 Survey participants' characteristics

\begin{tabular}{ll}
\hline Characteristics & $\mathbf{n}(\%)$ \\
\hline Male & $10(56)$ \\
First-year medical students & $18(100)$ \\
Involvement in DEPO diabetes-related training/ & $10(56)$ \\
volunteering & \\
DEE only & $2(20)$ \\
DEE + IVPT & $3(30)$ \\
IVPT + IVP & $3(30)$ \\
DEE + IVPT + IVP & $2(20)$ \\
\hline
\end{tabular}

Note: $n=18$.

Abbreviations: DEE, Diabetes Enrichment Elective; DEPO, Diabetes Education, Prevention, and Outreach; IVP, inpatient volunteering program; IVPT, inpatient volunteering program training. 
were compared with the results of those with no such training. Statistical analyses were performed by using SPSS Version 24 (IBM Corporation, Armonk, NY, USA). A Mann-Whitney $U$ test revealed a statistically significant difference in students' confidence in 4 of the 6 areas assessed favoring the group who participated in any form of DEPO DSMES training/ volunteering ( $\mathrm{U}=13-18, \mathrm{z}=-2.035-2.527, p<0.05$; Table 3$)$. A medium to large effect size was detected $(\mathrm{r}=0.48-0.59)$. The areas in which a significant improvement was seen included, students' confidence in assessing patients' understanding of diabetes, sick day diabetes management, and lifestyle modifications. There was no statistically significant difference in students' confidence in discussing monitoring/managing blood glucose or signs, symptoms, and treatment of hypoglycemia between the two groups. The relationship between training and confidence in discussing blood glucose monitoring and hypoglycemia was medium ( $\mathrm{r}=0.4$ and 0.35 , respectively).

\section{Discussion}

In this study, we assessed and compared the effectiveness of supplemental training on practical aspects of diabetes care and self-management on first-year medical students' confidence in addressing various aspects of DSMES with patients with diabetes. Through a survey, students who participated in any form of DSMES training/volunteer program reported statistically significant increased confidence in most assessed aspects of providing DSMES compared with those who did not participate in any training. The fact that disease-specific extracurricular education can be beneficial in medical training has been characterized in the literature. A previous study determined that an online bank of diabetes-based clinical cases could address junior doctors' confidence in managing patients with diabetes. ${ }^{24}$ In a study previously published by our group, it was demonstrated that a preclerkship Diabetes Enrichment Elective improved medical students' confidence in specific aspects of DSMES discussions with patients. ${ }^{22}$ The current study differs from our previous one in that it assesses the effectiveness of various training modalities in this area versus a single modality evaluated previously. Furthermore, the current study presents stronger methodology with using comparison with a control group versus the previous retrospective pre-/ post-course survey of same subjects. We also used a 5-point Likert scale versus a 4-point one used in the previous study to expand the results. Several other studies have shown positive results in implementing similar programs with pharmacy students. ${ }^{25-28}$ The results of the present study are consistent with those of previously published studies, which emphasize the potential benefits of implementing such programs. Our

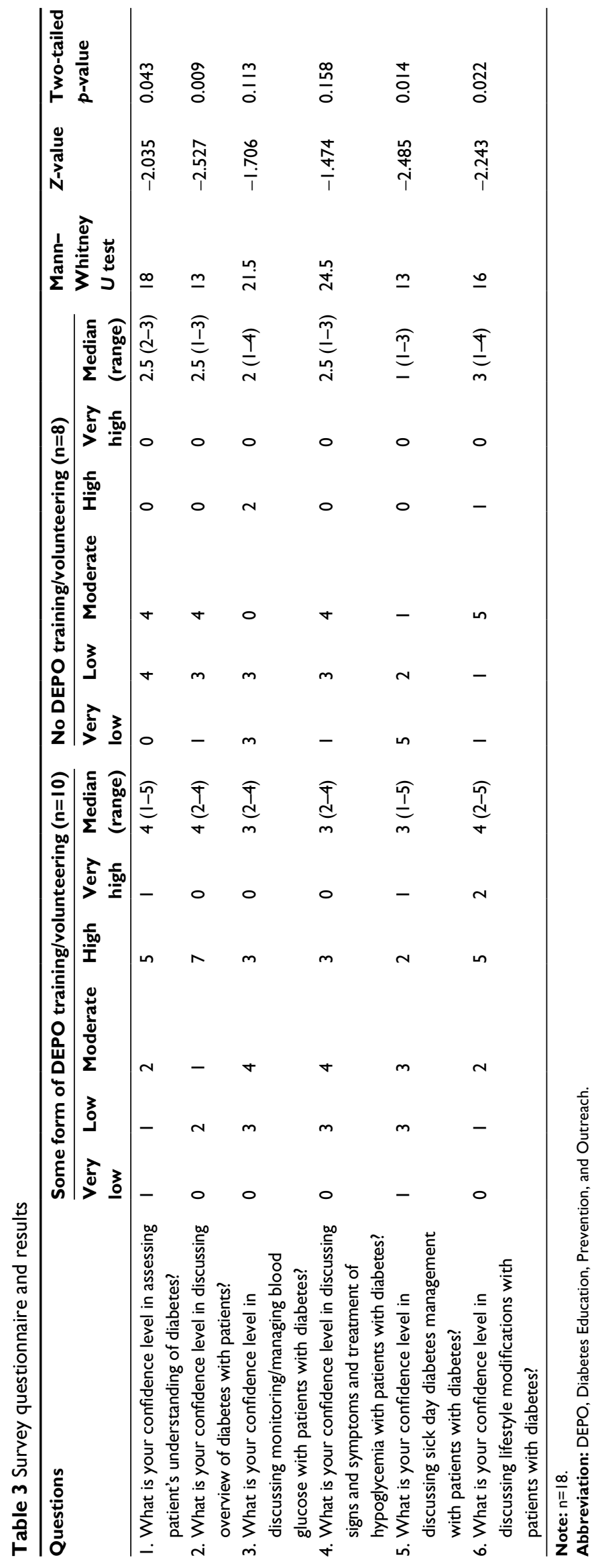


study differs from such studies in the population included and in the fundamental components of the educational interventions, including the direct inpatient experience provided to students allowing for patient interaction.

Furthermore, our study expands on these results by comparing specific supplementary educational modalities with the determination that the specific modality may not be critical to the overall benefit.

The results of the survey also indicated that despite the reported higher confidence in discussing management/monitoring of blood glucose or signs, symptoms, and treatment of hypoglycemia by those who received training, the difference between the two groups was not statistically significant. These results were not consistent with a previous study performed by our group, in which a diabetes enrichment elective improved medical students' confidence in addressing these topics. ${ }^{22}$ In the current study, a medium relationship was found between training and confidence in these areas with $r>0.3$. The small sample size reduces the test's power to detect a statistically significant difference between groups, if one exists.

A previous study has shown significant improvement in confidence and knowledge of doctors in training following the implementation of diabetes-specific education programs. ${ }^{29}$ Comparison of absolute effects, however, was not performed due to the inherent differences in measurements across the studies and use of nonvalidated surveys. Still, our results suggest that while educational intervention had a positive impact on medical students' confidence regarding the subject, the absolute impact may be a point of emphasis in future studies.

A limitation of this study was the small sample size and, hence, lack of ability to perform a subgroup analysis to compare the various training/volunteering modalities. Another limitation was that participants consisted entirely of first-year medical students, which may limit the generalizability of the results. Last, this study did not use a validated survey form, but rather a targeted form for the assessment of the effectiveness of the curriculum. However, the results of this study suggest the potential benefits of extracurricular disease-oriented education in medical training.

Future studies are required to evaluate the effect of DSMES provided by medical students on patient outcomes including hospital readmission.

\section{Conclusion}

This study suggests the beneficial impact of various diabetesspecific, DSMES-focused, preclerkship training modalities in forms of Diabetes Enrichment Elective, a 3-hour endocrinologist-led training session, and/or involvement in an inpatient DSMES volunteer program in supplementing medical students' education with practical knowledge while improving their confidence in providing such training/support to patients with diabetes.

\section{Disclosure}

The authors report no conflicts of interest in this work.

\section{References}

1. International Diabetes Federation. IDF Diabetes Atlas. 7th ed. Brussels: International Diabetes Federation; 2015. Available from: http://www. idf.org/diabetesatlas. Accessed May 11, 2017.

2. Centers for Disease Control and Prevention. National Diabetes Statistics Report: Estimates of Diabetes and Its Burden in the United States, 2014. Available from: https://www.cdc.gov/diabetes/pubs/statsreport14/ national-diabetes-report-web.pdf. Accessed May 23, 2016.

3. Statistics about Diabetes. Available from: http://www.diabetes.org/ diabetes-basics/statistics/. Accessed August 27, 2016.

4. Fazel MT, Pendergrass ML. Individualizing treatment of hyperglycemia in type 2 diabetes. JCOM. 2017;24(1):23-38.

5. Aro S, Kangas T, Reunanen A, Salinto M, Koivisto V. Hospital use among diabetic patients and the general population. Diabetes Care. 1994;17(11):1320-1329.

6. Fraze, T.K. (Thomson Reuters), Jiang, H.J. (AHRQ), and Burgess, J. (Thomson Reuters). Hospital Stays for Patients with Diabetes, 2008. HCUP Statistical Brief \#93. August 2010. Agency for Healthcare Research and Quality, Rockville, MD. Available from: http://www.hcupus.ahrq.gov/reports/statbriefs/sb93.pdf. Accessed September 13, 2017.

7. Centers for Disease Control and Prevention. National Diabetes Statistics Report, 2017. Available from: https://www.cdc.gov/diabetes/data/ statistics/statistics-report.html. Accessed August 5, 2017.

8. American Association of Diabetes Educators (AADE). Role of the diabetes educator in inpatient diabetes management. Diabetes Educ. 2017;43(1):28-33.

9. Beck J, Greenwood DA, Blanton L, et al. 2017 National Standards for Diabetes Self-management Education and Support. Diabetes Educ. Epub 2017 Jul 1.

10. Nettles AT. Patient education in the hospital. Diabetes Spectr. 2005;18(1):44-48

11. Healy SJ, Black D, Harris C, Lorenz A, Dungan KM. Inpatient diabetes education is associated with less frequent hospital readmission among patients with poor glycemic control. Diabetes Care. 2013;36(10):2960-2967.

12. Magee MF, Khan NH, Desale S, Nassar CM. Diabetes to Go: Knowledge- and competency-based hospital survival skills diabetes education program improves postdischarge medication adherence. Diabetes Educ. 2014;40(3):344-350.

13. Siddique H, Alam MM, Safi AW, Leong WB, Crowley K, Tahrani AA. In-patient diabetes care: the impact of diabetes outreach team on longterm glycaemic control. Int J Clin Pract. 2014;68(11):1333-1337.

14. Wexler DJ, Beauharnais CC, Regan S, Nathan DM, Cagliero E, Larkin ME. Impact of inpatient diabetes management, education, and improved discharge transition on glycemic control 12 months after discharge. Diabetes Res Clin Pract. 2012;98(2):249-256.

15. Buysman EK, Liu F, Hammer M, Langer J. Impact of medication adherence and persistence on clinical and economic outcomes in patients with type 2 diabetes treated with liraglutide: a retrospective cohort study. Adv Ther. 2015;32(4):341-355.

16. Suvarna HI, Moodithaya S, Sharma R. Metabolic and cardiovascular ageing indices in relation to glycated haemoglobin in healthy and diabetic subjects. Curr Aging Sci. Epub 2017 Feb 16.

17. Chopra I, Wilkins TL, Sambamoorthi U. Hospital length of stay and all-cause 30-day readmissions among high-risk Medicaid beneficiaries. J Hosp Med. 2016;11(4):283-288. 
18. Korytkowski MT, Koerbel GL, Kotagal L, Donihi A, DiNardo MM. Pilot trial of diabetes self-management education in the hospital setting. Prim Care Diabetes. 2014;8(3):187-194.

19. Shahla L, Vasudev R, Chitturi C, Rodriguez C, Paul N. Diabetes mellitus treatment-related medical knowledge among health care providers. Diabetes Metab Syndr. 2017;11(1):69-72.

20. George JT, Warriner D, McGrane DJ, et al. Lack of confidence among trainee doctors in the management of diabetes: the Trainees Own Perception of Delivery of Care (TOPDOC) Diabetes Study. QJM. 2011;104(9): 761-766.

21. Lansang MC, Harrell H. Knowledge on inpatient diabetes among fourth-year medical students. Diabetes Care. 2007;30(5):1088-1091.

22. Fazel MT, Fazel M, Bedrossian NL, Picazo F, Pendergrass ML. A student-implemented elective to improve medical student confidence in providing diabetes self-management support. Adv Med Educ Pract. 2016;7:567-570.

23. Haas L, Maryniuk M, Beck J, et al. National standards for diabetes self-management education and support. Diabetes Care. 2014; 37(Suppl 1):S144-S153.
24. Kelly NA, Brandom KG, Mattick KL. Improving preparedness of medical students and junior doctors to manage patients with diabetes. BMJ Open Diabetes Res Care. 2015;3(1):e000116.

25. Wongwiwatthananukit $\mathrm{S}, \mathrm{Z}$. for pharmacy students on providing diabetes care. Am J Pharm Educ. 2013;77(7):153.

26. Shrader S, Kavanagh K, Thompson A. A diabetes self-management education class taught by pharmacy students. Am J Pharm Educ. 2012;76(1):13.

27. Westberg SM, Bumgardner MA, Brown MC, Frueh J. Impact of an elective diabetes course on student pharmacists' skills and attitudes. Am J Pharm Educ. 2010;74(3):49.

28. Morello CM, Neighbors M, Luu L, Kobayashi S, Mutrux B, Best BM. Impact of a first-year student pharmacist diabetes self-care education program. Am J Pharm Educ. 2013;77(10):215.

29. Rajendran R, Kerry C, Round RM, et al. Impact of the Diabetes Inpatient Care and Education (DICE) project and the DICE Care Pathway on patient outcomes and trainee doctor's knowledge and confidence. Diabet Med. 2015;32(7):920-924.
Advances in Medical Education and Practice

\section{Publish your work in this journal}

Advances in Medical Education and Practice is an international, peerreviewed, open access journal that aims to present and publish research on Medical Education covering medical, dental, nursing and allied health care professional education. The journal covers undergraduate education, postgraduate training and continuing medical education

\section{Dovepress}

including emerging trends and innovative models linking education, research, and health care services. The manuscript management system is completely online and includes a very quick and fair peer-review system. Visit http://www.dovepress.com/testimonials.php to read real quotes from published authors.

Submit your manuscript here: http://www.dovepress.com/advances-in-medical-education-and-practice-journal 